\title{
Is intervention necessary after a first episode of acute idiopathic pancreatitis?
}

\author{
A B Ballinger, E Barnes, E M Alstead, P D Fairclough
}

\begin{abstract}
Acute idiopathic pancreatitis is a term used when no underlying cause has been identified on routine investigation. However, more specialised investigations may identify aetiological factors, biliary sludge and sphincter of Oddi dysfunction for example, in $38-72 \%$ of patients with recurrent episodes. Treatment of these abnormalities may prevent further episodes of pancreatitis. The aim of this study was to follow up and determine the outcome in patients with a first episode of idiopathic pancreatitis, and thus determine the need for further investigation and treatment in this group of patients. Thirty one patients with a single episode of idiopathic pancreatitis were studied who had no specialised investigations or specific treatment. During a median follow up of 36 months only one patient has had recurrent pancreatitis. Two patients experienced a single episode of unexplained abdominal pain; serum amylase, liver biochemistry, and abdominal ultrasound were all normal and the pain resolved within 48 hours. In conclusion, in the medium term, the prognosis is good after a first episode of idiopathic pancreatitis and specialised investigation is unnecessary.

(Gut 1996; 38: 293-295)
\end{abstract}

Keywords: idiopathic pancreatitis, recurrence.

Acute pancreatitis is a common emergency with considerable morbidity and a mortality of about $7 \% .{ }^{1}$ In view of this, an underlying cause is usually sought so that appropriate treatment may be given and recurrent episodes prevented. In Europe and the United States, gall stones and alcohol excess are the most common causes, accounting for $64-80 \%$ of cases. Drugs, metabolic abnormalities, viral infections, trauma, and a variety of other rarer causes are implicated in up to $10 \%$ of cases. Finally in $10-20 \%$ of cases no abnormality is detected despite a careful history, biochemical screen, and ultrasound or computed tomography of the abdomen. ${ }^{2-4}$ These patients are conventionally classified as having acute idiopathic pancreatitis. Recent studies have reported, however, that with more specialised and invasive investigations possible aetiological factors are identified in $38-73 \%$ of these cases. $^{5-7}$ The causes include sphincter of Oddi dysfunction, biliary sludge, pancreas divisum, and pancreatic duct abnormalities. Specific treatment of these conditions reduced the recurrence rate compared with an untreated group. However, these studies recruited either only patients with recurrent episodes of pancreatitis $^{5}$ or a mixed group consisting of first attenders and patients with recurrent episodes. ${ }^{67}$ This differentiation is important as the aetiology and prognosis may not be the same in these two groups.

The purpose of this study was to follow up patients with a first episode of idiopathic pancreatitis who did not undergo specialised investigations or intervention. The results suggest that within three years of the index episode a second episode is rare and specialised investigation and intervention are thus unnecessary.

\section{Methods}

Patients with acute pancreatitis treated at the Homerton Hospital, St Bartholomew's Hospital, and Whipps Cross Hospital between January 1989 and January 1994 were identified from hospital coding and review of 1469 consecutive endoscopic retrograde cholangiopancreatography (ERCP) records. The purpose of reviewing the ERCP records was to identify any patients with acute pancreatitis who may have been coded incorrectly and would therefore not be identified from coding. Although this would detect only those patients who had an ERCP, this was the only source available to identify incorrectly coded patients. In fact, review of the ERCP records only picked up two additional cases of acute pancreatitis not identified from hospital coding.

The hospital records of all possible cases were reviewed and a definite diagnosis of acute pancreatitis was made if the serum amylase was more than five times the upper limit of the hospital reference range (in the absence of other conditions causing hyperamylasaemia, for example, perforated peptic ulcer) or if computed tomography showed changes of acute pancreatitis. Patients were considered to have acute idiopathic pancreatitis if they had no history of alcohol abuse (defined as average daily consumption of more than three units per day), no gall bladder or common bile duct calculi on ultrasound examination, no recent intake of drugs reported to cause acute pancreatitis and no hypertriglyceridaemia or hypercalcaemia. In addition, patients with any of the rarer causes of acute pancreatitis and patients who had previously had a cholecystectomy were not included in the study. All patients with a first episode of acute pancreatitis who fulfilled these exclusion criteria were recruited for the study. Follow up was recorded by review of the hospital records and by a questionnaire (Figure) sent to both the patients and their general practitioner. During 
Dear Mrs Jones

I am writing to ask if you would help with a small study that we are performing at St Bartholomew's Hospital. As you know, you were admitted in May 1991 with pancreatitis (inflammation of the pancreas gland). We are attempting to obtain long-term follow up on patients admitted with this condition. I would be grateful if you would answer the following questions and send your reply in the stamped addressed envelope provided.

1. Have you had any abdominal (tummy) pains since the attack of pancreatitis? If

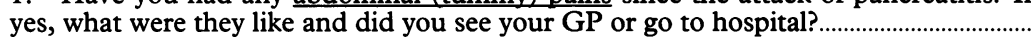

2. Have you had any further attacks of pancreatitis? If yes, what hospital were you admitted to?

3. Have you had any operations since the attack of pancreatitis? If yes, what operation?..........

4. Please list any other illnesses that you have had since the attack of pancreatitis?

Thank you for your help

Yours sincerely

An example of the follow up questionnaire sent to the patient. The questionnaire (not shown) sent to the general practitioner asked the same types of questions and the wording changed appropriately.

the follow up period, at least two questionnaires were sent to each patient specifically designed to discover if there had been any possible further episodes of pancreatitis or symptoms to suggest gall stone disease.

\section{Results}

Thirty one patients ( $15 \mathrm{men}$ ) were identified as having a first episode of acute idiopathic pancreatitis. The mean age of the study group at the time of the acute illness was 61.7 years (range 24-92). The serum amylase was five times the upper limit of normal in all except two patients in whom computed tomography showed focal pancreatitis. All patients satisfied the exclusion criteria; triglycerides were measured in 18 patients and found to be normal in all. The inclusion criteria for this study did not include a normal ERCP, however, an ERCP had been performed in 20 patients and no aetiological factor identified. It is not the policy of the hospitals in this study to routinely perform ERCP on all patients with idiopathic pancreatitis and the decision to perform ERCP was made by the consultant in charge of the patient. At ERCP, no patient had a sphincterotomy or sphincter manometry. There were no complications of ERCP in this group of patients. There was no significant difference in age between the group of patients who had ERCP (58 years (range 24-76)) and those who did not (69 (range 39-92)). Serum alkaline phosphatase was significantly higher (Mann-Whitney test, $p=0.0056$ ) in the ERCP patients (median 158, interquartile range 126-318 IU/l) compared with the group who did not have an ERCP (median 69, 60-94). Serum aspartate aminotransferase (ERCP, 40 (17-78 IU//); no ERCP, 38 (22-164)) and serum bilirubin (ERCP, 13 (10.5-73.5 $\mu \mathrm{mol} / \mathrm{l})$; no ERCP, 15 (11-17)) was the same in both groups.

Of the 31 patients studied, two died during the acute illness from multi-organ failure and follow up is incomplete in two patients. One of the patients who died had a postmortem examination, which showed a necrotic pancreas and pulmonary oedema; no gall stones or other possible aetiological factor of acute pancreatitis was identified. Median follow up in the remaining 27 patients is 36 months (interquartile range 24-54). Only one patient has had a further episode of acute pancreatitis occurring nine months after the first episode. Investigations on the second admission including serum triglycerides, abdominal computed tomography, and ERCP did not reveal a cause and he remained well in the 15 month follow up. One patient has died in the follow up period of a myocardial infarction. Seven patients have had episodes of abdominal pain. Two of these were diagnosed as having a peptic ulcer (one at gastroscopy and one on barium meal examination) and the pain has resolved with appropriate treatment. Two patients were seen in the accident and emergency department and investigations including serum amylase, liver biochemistry, and abdominal ultrasound were all found to be normal. The pain resolved in less than 48 hours with no firm diagnosis being made and no recurrence of symptoms since. The remaining three patients had mild episodes of dyspepsia, which have not necessitated a visit to the hospital or general practitioner. No patients have developed jaundice or had a cholecystectomy.

\section{Discussion}

Biliary sludge, defined as a suspension of cholesterol monohydrate crystals or calcium bilirubinate granules, has been implicated in the pathogenesis of acute idiopathic pancreatitis. Microscopic examination of bile, obtained by duodenal intubation, has identified sludge in $67-74 \%$ of patients after an acute episode of idiopathic pancreatitis. ${ }^{67}$ Lee et al showed that recurrent episodes of pancreatitis occurred in eight of $11(73 \%)$ untreated patients with sludge. In contrast only one of 10 patients treated by cholecystectomy or endoscopic sphincterotomy developed recurrent pancreatitis. ${ }^{7}$ In a similar study, Ros et al showed that a high proportion of patients with sludge developed gall bladder stones on follow up examination. ${ }^{6}$ Recurrent pancreatitis occurred in 12 of those patients $(67 \%)$ in whom cholecystectomy was not performed or in whom gall stones persisted despite treatment with ursodeoxycholic acid. In both of these studies treatment was not randomised and it is unclear why some patients were assigned to treatment and others not. In addition these studies recruited both patients with a first episode of pancreatitis and patients with recurrent episodes. Other studies have recruited only those patients with recurrent episodes of idiopathic pancreatitis and identified possible aetiological factors in $31-46 \%$ of patients. ${ }^{5} 89$ These included pancreas divisum, cholelithiasis, choledochocoele, papillary tumour, and sphincter of Oddi dysfunction.

Our study has examined the outcome only in those patients with a first episode of idiopathic pancreatitis. No patient has had a sphincterotomy, cholecystectomy or treatment 
with ursodeoxycholic acid in the follow up period. Over a follow up period of three years, only one patient $(4 \%)$ has had a further episode of pancreatitis. A previous study has shown that some patients with biliary sludge develop gall stones and therefore we were interested to know if our patients developed symptoms of gall stone disease; no patients have developed cholecystitis, biliary colic or jaundice, and no patient has had a cholecystectomy. Gall bladder ultrasound has not been performed during the follow up period and therefore we do not know if any of the patients have developed asymptomatic gall stones.

Although this is a retrospective study, follow up was complete in all but two patients. In these two patients the hospital records could not be found and the general practitioner or patient questionnaire was not returned. The follow up questionnaire was specifically designed to detect if there had been recurrent episodes of pancreatitis or symptoms of cholelithiasis. Patients may have forgotten, and therefore not reported, a mild short lived episode of abdominal pain. Pancreatitis, cholecystitis, and biliary colic usually cause pain, however, which is severe enough to prompt a visit to the general practitioner or hospital. We would therefore expect that even if symptoms were not reported on the patient questionnaire, they would be reported by the general practitioner. In addition, to aid recall, a questionnaire was sent out twice in the follow up period.

In conclusion, we have found that the incidence of second episodes of pancreatitis is low, at least in the short to medium term. In this group of patients the risk of complications from ERCP are greater than the risks of a second episode. We therefore feel that the morbidity and mortality of specialised investigation after a first episode of acute idiopathic pancreatitis (defined as those patients with a normal ultrasound other than pancreatitis, no history of alcohol misuse, and no other obvious cause of pancreatitis) probably outweighs the benefits. We will continue to follow up this cohort of patients.

Supported by the National Association for Crohn's and Colitis, the Magdalene Hughes Endowment Fund, and the Ernie Whitelaw Research Fund. ABB is supported by the Joint Research Board of St Bartholomew's Hospital.

This work was presented to the 1995 Spring meeting of the This work was presented to the 1995 Spring meeting of the
British Society of Gastroenterology and the 1995 annual meeting of the American Gastroenterological Association.

1 Ranson JHC. Acute pancreatitis: surgical management. In: Go VLW, Dimagno EP, Gardner JD, Lebenthal E, Reber
HA, Scheele GA, eds. The pancreas: biology, pathobiology HA, Scheele GA, eds. The pancreas: biology, pathobio
and disease. New York: Raven Press, 1993: 637-48.

2 Neoptolemos JP, Hall AW, Finlay DF, Berry JM, CarrLocke DL, Fossard DP. The urgent diagnosis of gallstones in acute pancreatitis: a prospective study of three methods. Br $\mathcal{F}$ Surg 1984; 71: 230-3.

3 Imrie CW, Whyte AS. A prospective study of acute pancreatitis. Br $\mathcal{F}$ Surg 1975; 62: 490-4

4 Clavien P, Robert J, Meyer P, Borst F, Hauser H, Hermann $\mathrm{F}$, et al. Acute pancreatitis and normoamylasemia. Ann Surg 1989; 210: 614-20.

5 Venu RP, Geenen JE, Hogan W, Stone J, Johnson GK, Soergel K. Idiopathic recurrent pancreatitis. Dig Dis Sci 1989; 34: 56-60.

6 Ros E, Navarro S, Bru C, Garcia-Puges A, Valderrama R. Occult microlithiasis in 'idiopathic' acute pancreatitis: prevention of relapses by cholecystectomy or ursodeoxycholic acid therapy. Gastroenterology 1991; 101: 1701-9.

7 Lee SP, Nicholls JF, Park HZ. Biliary sludge as a cause of acute pancreatitis. $N$ Engl f Med 1992; 326: 589-93.

8 Cooperman M, Ferrara JJ, Carey LC, Thomas FB, Martin EW Jr, Fromkes JJ. Idiopathic acute pancreatitis: The value of endoscopic retrograde cholangiopancreatography. Surgery 1981; 90: 666-70.

9 Feller ER. Endoscopic retrograde cholangiopancreatography in the diagnosis of unexplained pancreatitis. Ann Intern Med 1984; 144: 1797-9. 